\title{
Demografietarifverträge - Genese, Wirkungen und Perspektiven eines Gestaltungskonzeptes
}

\author{
W. Anlauft und W. Feldes
}

\section{Abstract}

Wie ein langes und intensives Arbeitsleben für älter werdende Beschäftigte gesundheitsgerecht gestaltet werden kann, ist eine seit Jahrzehnten tarifund arbeitspolitisch nicht zufriedenstellend gelöste Frage. Auch für die Zukunfts- und Entwicklungsperspektiven Älterer in der digitalen Transformation sind daher Vorkehrungen zu treffen: es kommt auf strategische Personalplanung, alternsgerechte Arbeitsgestaltung, frühzeitige Technikund Qualifikationsgestaltung und konfliktbereite Interessenvertretungen an.

\section{Einleitung}

Im Rückblick auf die Anfänge demografieorientierter Arbeitspolitik in den 90er-Jahren des letzten Jahrhunderts fällt auf, dass die Gewerkschaften in den darauffolgenden beiden Jahrzehnten keine ernsthaften Versuche unternommen haben, Demografieverträge für alle von ihnen vertretenen Beschäftigten abzuschließen. Obwohl die systemische Bedeutung des gesellschaftlichen Altersstrukturwandels schon damals als unumstritten galt (Volkholz 1993), haben die Gewerkschaften den Tarifvertrag - ihr wichtigstes Gestaltungsinstrument - im Gestaltungsfeld Demografie mit wenigen Ausnahmen zurückhaltend nur in Teilbranchen zum Einsatz gebracht und nicht ins Zentrum ihrer Tarifpolitik gestellt. Die sozialwissenschaftliche Beforschung dieser überschaubaren Zahl von demografiezentrierten Branchenverträgen kommt daher zu dem wenig überraschenden Befund eines geringen Umsetzungs- und Verbreitungsgrades demografiesensibler Arbeitsgestaltungs- und Personalentwicklungsmaßnahmen in den Unternehmen. Insbesondere bei altersbezogenen Maßnahmen sind kaum mehr als 30 Prozent der Betrieb gestaltungsaktiv (Tullius u.a. 2012).

Damit interessiert sich dieser Beitrag am Beispiel der Demografieverträge der IG Metall für die Gründe dieser defensiven Regulierungspolitik. 
Wir tragen Erklärungen für die begrenzte Reichweite und Umsetzungstiefe der Regelungen zusammen. In unserem Fazit vertreten wir die Auffassung, dass eine Renaissance der Tarifierung alter(n)sgerechter Arbeits- und Personalpolitik trotz zunehmender Demografiedynamik wenig wahrscheinlich ist. Gleichwohl ist auszuloten, wie die Beschäftigungssicherungs- und Arbeitsgestaltungsinteressen des immer noch wachsenden Anteils älterer Beschäftigter im Kontext der digitalen Transformation zielführender als bisher zur Geltung kommen können.

\section{Eine Regulierungsgeschichte in zwei Phasen}

Die Geschichte der Demografieverträge ist schnell erzählt. Sie beginnt mit der Anhebung der Altersgrenzen seit 1992 im Kontext der neoliberalen Demontage des Sozialsystems unter der Regierung Kohl. Sie setzt sich fort mit der Rentenpolitik der rot-grünen Bundesregierung ab 1999', die durch die radikale Begrenzung von Optionen eines vorzeitigen Erwerbsausstieges und durch die Absenkung des Rentenniveaus gekennzeichnet ist. Diese Demontage der lebensstandardsichernden Rentenversicherung und die letztlich erfolglosen Proteste von Gewerkschaften, Kirchen und Sozialverbänden gegen den Abbau sozialer Leistungen markieren in der Mitte der 2000er-Jahre schließlich die entscheidenden Auslöser für das tarifvertragliche Projekt der Regulierung von Industrie- und Dienstleistungssektoren mit Demografieverträgen.

Während sich also mit dem Aufknüpfen der sozialstaatlichen Sicherungsnetze der korporatistische Konsens zwischen Gewerkschaften, Staat und Unternehmen (»Modell Deutschland«) über einen frühen und sozialverträglichen Altersübergang auflöst, lassen sich im Organisationsbereich der IG Metall in der Folge zwei Regulierungsphasen beobachten, in denen zugleich die Vertariflichung sozialer Sicherung (Fröhler und Fehmel 2018) zum Ausdruck kommt. Neben der Beteiligung gewerkschaftlich Aktiver an einer Vielzahl öffentlich geförderter betrieblicher Alternsmanagementprojekte (Prognos AG 2010) entsteht mit dem 2006 abgeschlossenen Tarifvertrag zum demografischen Wandel in der Eisen- und Stahlindustrie die Blaupause zur tarifpolitischen Annäherung an das Gestaltungsfeld Demografie.

Der Tarifvertrag knüpft dabei - unter dem Eindruck des sozialpolitischen Rückzugs des Staates - an die gewerkschaftliche Tradition gesellschaftspolitischer Gestaltung über tarifvertragliche Impulse an (Katenkamp u.a. 2012) und entsteht nicht zufällig in zwei Wirtschaftsbranchen, in denen die mit Arbeitsbelastungen verbundenen Personalprobleme tra- 
ditionell deutlicher und früher sichtbar werden als in anderen Wirtschaftszweigen. Als ein neuer Typus von Prozessvereinbarung ist er darauf ausgerichtet, datengestützte Gestaltungsprozesse zu initiieren (Belastungsabbau, Gesundheitsförderung, Altersteilzeit, Weiterbildung), die sich in alternsund altersgerechten Entlastungsmaßnahmen konkretisieren sollen. Durch die Gestaltung körperlich und/oder psychisch entlastender Arbeitsbedingungen erhoffte man sich, Beschäftigungsalternativen im internen Arbeitsmarkt kreieren zu können, die einen längeren Verbleib im Unternehmen, Fachkräftesicherung, aber auch Vorteile bei der Personalbeschaffung erbringen würden. Die Einrichtung von Demografiefonds blieb jedoch noch ganz dem alten personalpolitischen Paradigma des frühen Altersübergangs verhaftet. Auch nach dem Wegfall der gesetzlichen Regelungen zur Altersteilzeit sollten die fondsfinanzierten Aufstockungsbeträge zu den Entgelten in der Altersteilzeit weiterhin die Türen zur vorzeitigen Beendigung des Arbeitslebens offenhalten.

Die tarifvertragliche Implementierung von betrieblichen Alternsmanagementmaßnahmen tritt nach der Finanzmarktkrise von 2009 in eine zweite Phase. Es kommt jedoch nicht zu einer Ausdehnung der Tarifierung des Altersstrukturwandels auf die Metall- und Elektroindustrie. Dort wird die Alters- und Fachkräfteproblematik mit Spezialregelungen zum flexiblen Übergang in die Rente und zur Qualifizierung abgefangen. Einschlägige Demografievereinbarungen entstehen ab 2012 noch im KfZHandwerk Niedersachsen, in der Holz- und Kunststoffindustrie (2016/17) und in der Schreib- und Zeichengeräteindustrie (2016). Hauptziel ist die Einführung des in der Weiterverarbeitung üblichen Sicherungsniveaus bei der Altersteilzeit. Materieller Regelungsgehalt ist wie schon zuvor in der Stahl- und Eisenindustrie die Kombination von Maßnahmen einer demografieorientierten Personalpolitik mit Möglichkeiten des flexiblen Altersübergangs. Der Abschluss eines Demografievertrages 2013 in der Textilund Bekleidungsbranche scheitert unterdessen. Die sog. Gute-Arbeit-Kampagne der IG Metall (2011/ 2012) bezieht sich zwar auf den gesellschaftlichen Demografiediskurs und erreicht auch in einigen Fällen den Abschluss betrieblicher Demografievereinbarungen. Ihr Anknüpfen an das Demografienarrativ und das Skandalisieren arbeitspolitischer Missstände verfolgte jedoch in der Hauptsache den Zweck der betrieblichen Mobilisierung für die rentenpolitischen Forderungen der IG Metall. 


\section{Regelungs- und Gestaltungsbilanz des Tarifkonzeptes}

Eine differenzierte Analyse der Wirkungen des Tarifkonzeptes für die unterschiedlichen Branchen im Organisationsbereich der IG Metall kann sich nicht auf repräsentative belastbare quantitative oder qualitative Studien stützen. Es gibt keine einschlägigen empirischen Untersuchungen oder systematisch aufbereitete betriebliche Fallstudien. Auch die einzelnen Tarifverträge sind - wie oben skizziert - in ihrer Anlage und Zielsetzung heterogen und vielfältig und umfassen ein äußerst unterschiedliches Bündel an Regelungen: Tarifvertrag zur betrieblichen Altersteilzeit, Tarifvertrag als ergebnisoffene Prozessvereinbarung (Analyse, Ableitung von Gestaltungsmaßnahmen, Wirksamkeitskontrollen), Fondslösungen, in denen Möglichkeiten zum vorzeitigen Ausscheiden aus dem Erwerbsleben unterstützt werden oder verhaltens- und verhältnisorientierte Gestaltungsmaßnahmen für eine gesundes Älterwerden im Betrieb finanziert werden oder Kampagnen, in denen Elemente einer alter(n)sgerechten Arbeitsgestaltung verknüpft werden mit einer politischen Mobilisierung für eine andere Rentenpolitik.

Bilanzierende Betrachtungen zur Wirksamkeit und zum Wirkungsgrad von »Tarifverträgen Demografie« müssen sich daher in erster Linie auf persönliche Einschätzungen der Betrachter stützen. Diese werden angereichert um betriebliche oder branchenbezogene Berichte und Erfahrungen einschlägiger Akteure aus Unternehmen (Betriebsräte, Personalmanagement, Gesundheitsakteure, Beschäftigte), Verbänden und Gewerkschaften.

Darüber hinaus können wir jedoch auch auf Sekundärdaten als Wirkungsindikatoren zurückgreifen. Sekundärdaten etwa zum Ausmaß der betrieblichen Maßnahmen zur alter(n)sgerechten Arbeitsgestaltung (IAB Betriebspanel) oder zur Entwicklung der Arbeitsbedingungen (Erwerbstätigenbefragungen BIBB/BAuA) stellen - mit gewissen Einschränkungen ${ }^{2}$ wichtige und brauchbare Hinweise zu den Wirkungen des arbeitspolitischen Demografiediskurses dar. So kommen die Erwerbstätigenbefragungen von BiBB/BAuA 2006-2018 zu dem Ergebnis, dass längeres Arbeiten unter alternskritischen Bedingungen dazu führt, dass Mitarbeiter*innen ihren Gesundheitszustand als deutlich schlechter empfinden. ${ }^{4}$ Hinzu kommt, dass mit steigendem Alter, die Arbeitnehmer"innen diese alter $(\mathrm{n})$ skritischen Arbeitsbedingungen als deutlich belastender einstufen (Klippert 2014). 
Tabelle 1: Ausmaß körperlicher und psychischer Fehlbeanspruchungen

\begin{tabular}{|lrrr|}
\hline körperliche Faktoren & $\mathbf{2 0 0 6}$ & $\mathbf{2 0 1 2}$ & $\mathbf{2 0 1 8}$ \\
\hline Arbeiten im Stehen & 56,4 & 54,4 & 53,5 \\
Arbeiten im Sitzen & k.A. & k.A. & 52,9 \\
Arbeiten mit den Händen & k.A. & 41,8 & 38,7 \\
Zwangshaltungen & 14,3 & 16,6 & 16,6 \\
Heben und Tragen von schweren Lasten & 22,8 & 22,3 & 22,6 \\
Erschütterungen, Stöße und Schwingungen & 4,6 & 4,3 & k.A. \\
Lärm & 23,9 & 24,0 & 25,4 \\
grelles Licht, schlechte Beleuchtung & 9,3 & 9,0 & 9,0 \\
gefährliche Stoffe, Strahlung & 6,8 & 10,2 & k.A. \\
Mikroorganismen & 7,5 & 9,2 & 12,8 \\
Rauch, Gase, Staub, Dämpfe & 13,9 & 12,2 & 12,2 \\
Hitze, Kälte, Feuchtigkeit, Zugluft & 21,2 & 19,6 & 20,0 \\
Öl, Fett, Schmutz, Dreck & 17,6 & 16,3 & 17,2 \\
Arbeiten im Freien & k.A. & k.A. & 12,8 \\
\hline
\end{tabular}

\begin{tabular}{|lrrr|}
\hline psychische Faktoren & $\mathbf{2 0 0 6}$ & $\mathbf{2 0 1 2}$ & $\mathbf{2 0 1 8}$ \\
\hline schwer, nach der Arbeit abzuschalten & k.A. & k.A. & 21,9 \\
gefühlsmäßig belastende Situation & 12,1 & 11,8 & 12,2 \\
Konfrontation mit neuen Aufgaben & 39,1 & 40,1 & 40,1 \\
Verfahren verbessern/ neue ausprobieren & 27,8 & 27,6 & 29,2 \\
Arbeitsdurchführung detailliert vorgeschrieben & 22,9 & 24,8 & 24,9 \\
Arbeitsgang wiederholt sich & 51,4 & 48,4 & 45,5 \\
nicht alle notwendigen Informationen & 8,3 & 8,3 & 11,1 \\
nicht rechtzeitig informiert & 13,5 & 14,1 & 16,1 \\
nicht Beherrschtes wird verlangt & 8,8 & 7,6 & 8,2 \\
Störungen/ Unterbrechungen & 46,1 & 42,8 & 44,7 \\
Mindestleistung, Zeit vorgegeben & 31,1 & 29,7 & 29,2 \\
bis an die Grenze der Leistungsfähigkeit & 17,0 & 16,6 & 16,2 \\
schnell arbeiten müssen & 44,1 & 38,6 & 33,9 \\
starker Termin/-Leistungsdruck & 53,5 & 52,0 & 47,9 \\
verschiedene Arbeiten gleichzeitig & 58,7 & 58,7 & 60,9 \\
\hline
\end{tabular}

(Angaben in \% aller Befragten mit Antwort häufig)

Quelle: (eigene Darstellung auf Basis der BiBB/BAuA Erwerbstätigenbefragungen 2006, 2012 und 2018, Erwerbstätige mit mindestens 10 Std. wöchentlich, Stichprobe umfasst rund 20.000 Befragte) 
Auffallend ist, wie wenig sich die Ergebnisse der Befragungen unterscheiden. Anders ausgedrückt: Arbeitnehmer*innen beurteilen die Arbeitsbedingungen im Durchschnitt 2018 ähnlich wie im Jahr 2006. Zu erwarten wäre gewesen, dass angesichts der erheblichen Veränderungen in der Arbeitswelt und einer Reihe von Maßnahmen alterssensibler Arbeitsgestaltung in den letzten zwölf Jahren die Veränderungen deutlich höher hätten ausfallen müssen. Dies ist jedoch bei der Gesamtbetrachtung nicht der Fall. Ein ähnliches Bild zeichnet sich bei dem Ausmaß der arbeitsbedingten Ressourcen ab. Die Veränderungen zwischen 2006 und 2018 sind ebenfalls nur geringfügig. Eine Aufklärung über diesen Sachverhalt sollte weiteren Untersuchungen und Analysen vorbehalten sein.

Tabelle 2: Ausmaß der Ressourcen, die in der Arbeit vorhanden sind

\begin{tabular}{|lccr|}
\hline & $\mathbf{2 0 0 6}$ & $\mathbf{2 0 1 2}$ & $\mathbf{2 0 1 8}$ \\
\hline eigene Entscheidung, wann Pause & 55,9 & 57,6 & 63,1 \\
Einfluss auf Arbeitsmenge & 34,8 & 34,9 & 31,6 \\
Arbeit selbst planen & 69,7 & 68,9 & 65,5 \\
Gefühl, Tätigkeit ist wichtig & 79,1 & 77,8 & 77,6 \\
Lob/Anerkennung von Vorgesetzten & k.A. & k.A. & 32,5 \\
Unterstützung von Vorgesetzten & 58,4 & 59,0 & 58,9 \\
Unterstützung von Kollegen & 78,8 & 79,7 & 79,1 \\
gute Zusammenarbeit mit den Kolle- & & & \\
gen & 87,4 & 88,1 & 86,7 \\
Teil einer Gemeinschaft & 77,9 & 79,8 & 79,7 \\
\hline
\end{tabular}

(Angaben in \% der Befragten mit Antwort häufig)

Quelle: (eigene Darstellung auf Basis der BiBB/BAuA Erwerbstätigenbefragungen 2006, 2012 und 2018, Erwerbstätige mit mindestens 10 Std. wöchentlich, Stichprobe umfasst rund 20.000 Befragte)

Ernüchternd ist ebenfalls der Blick auf die Ergebnisse des IAB Betriebspanels von 2006 bis 2015. Trotz der deutlich steigenden Anzahl älterer Beschäftigter hat sich das Ausmaß der Maßnahmen für den Erhalt der Beschäftigungsfähigkeit Älterer kaum verändert. Rückläufig und zwar von $10 \%$ auf $5 \%$ der befragten Betriebe sind dagegen Maßnahmen im Bereich der Altersteilzeit. 
Tabelle 3: Anteil der Betriebe mit Maßnahmen für ältere Beschäftigte im Zeitverlauf

\begin{tabular}{|l|c|c|c|c|}
\hline & $\mathbf{2 0 0 6}$ & $\mathbf{2 0 0 8}$ & $\mathbf{2 0 1 1}$ & $\mathbf{2 0 1 5}$ \\
\hline Altersteilzeit & 10,0 & 9,0 & 8,0 & 5,0 \\
\hline Besondere Ausstattung der Arbeitsplätze & 1,0 & 2,0 & 2,0 & 3,0 \\
\hline $\begin{array}{l}\text { Individuelle Anpassung der Leistungs-/ } \\
\text { Arbeitsanforderungen }\end{array}$ & 2,0 & 2,0 & 4,0 & 5,0 \\
\hline $\begin{array}{l}\text { Altersgemischte Besetzung von Arbeits- } \\
\text { gruppen }\end{array}$ & 5,0 & 5,0 & 6,0 & 5,0 \\
\hline $\begin{array}{l}\text { Einbeziehung Älterer in betriebliche } \\
\text { Weiterbildung }\end{array}$ & 6,0 & 6,0 & 9,0 & 7,0 \\
\hline Spezielle Weiterbildung für Ältere & 1,0 & 1,0 & 1,0 & 1,0 \\
\hline $\begin{array}{l}\text { Einbeziehung Älterer in Maßnahmen } \\
\text { zur Gesundheitsförderung }\end{array}$ & k.A. & k.A. & 4,0 & 4,0 \\
\hline Andere Maßnahmen für Ältere & 1,0 & 1,0 & 1,0 & 1,0 \\
\hline Mindestens eine Maßnahme & 17,0 & 17,0 & 18,0 & 17,0 \\
\hline
\end{tabular}

(Angaben in \%, betrifft nur Betriebe mit älteren Arbeitnehmer*innen)

Quelle: IAB Betriebspanel 2006, 2008, 2011 und 2016

Ein erstes Zwischenfazit zur Altersgerechtigkeit von Tätigkeitsanforderungen besagt also: Der Anteil älterer Beschäftigter ist in den letzten Jahren sowohl in absoluten Zahlen als auch bezogen auf ihre Erwerbsquote deutlich gestiegen. Die Erwerbstätigenbefragungen von BiBB/BAuA verdeutlichen ein nach wie vor hohes Ausmaß alternskritischer Fehlbeanspruchungen in der Arbeitswelt. Das IAB Betriebspanel weist in absoluten Zahlen betrachtet nur einen geringfügigen Anstieg bei der ergonomischen Gestaltung der Arbeitsplätze und der individuellen Anpassung der Leistungsanforderungen aus. Das Ausmaß der Altersteilzeit ist dagegen im Ergebnis der gesetzlichen Veränderungen rückläufig.

Insgesamt betrachtet ergibt sich trotz 20-jährigem Demografiediskurs ein annähernd gleichbleibendes Ausmaß von betrieblichen Maßnahmen zum Erhalt der Beschäftigungsfähigkeit einer wachsenden Anzahl von Betrieben mit älteren Arbeitnehmer*innen.

Gilt dieser ernüchternde Befund ebenso für Betriebe aus den Branchen mit Demografievertrag, oder sind die Beschäftigten dort in einer besseren Situation? 
Aus unserer Sicht unzweifelhaft erfolgreich waren die Akteure beim Agenda-Setting, also bei der Übersetzung und Verwandlung des gesellschaftlich angeheizten Problemdiskurses (»Das Methusalem-Komplott «) in einen betrieblichen Gestaltungsdiskurs. In den Betrieben, in denen im Anschluss an die Demografieanalysen Arbeitsbedingungen altersgerechter und Altersteilzeit weiter möglich waren, konnten sich Beschäftigte darin bestätigt sehen, dass ihre Erwartungen nach einer komplikationslosen Exitstrategie aus einem langen und verschleißträchtigen Erwerbsleben wahrgenommen wurden. Gelungen ist auch die Sensibilisierung von Unternehmen für eine demografieorientierte Personalarbeit. Auch in den Betriebsratsgremien und bei den Schwerbehindertenvertretungen haben die Tarifverträge Spuren im betriebspolitischen Mindset hinterlassen.

Eine altersdifferenzierte Betriebspolitik hat sich dagegen nur selten und nur mit Mühe herausgebildet (Katenkamp 2012). Die Aktivitäten, die aus prozessorientierten Vereinbarungen resultieren, zeigen eine Vielfalt an Projekten und Einzelmaßnahmen, wie z.B. ergonomische Gestaltung von Arbeitsplätzen, persönlichkeitsförderliche Tätigkeitsgestaltung durch Anreicherung und Rotation, verbesserte Schichtplan- und Arbeitszeitgestaltung, Maßnahmen zur Begrenzung von Leistungsanforderungen und Reduzierung entgrenzter Arbeit, altersgemischte Teams, frühzeitiger Knowhow Transfer als Bestandteil der Nachfolgeplanung oder Projekte zur demografiesensiblen Führungsarbeit.

Diese Vorhaben waren in aller Regel als Projekte auf Zeit angelegt. Die meisten Projekte haben dabei das Stadium des Modellprojektes nicht verlassen. Es fehlte an einer erfolgreichen Überführung in eine betriebliche Regelpraxis, die in sich kohärent ist und über einen längeren Zeitraum versehen als kontinuierlicher Entwicklungs- und Gestaltungsprozess betrieben wurde. Daher sind die positiven Wirkungen dieser Tarifverträge und Kampagnen durchaus sichtbar, sie sind aber in den meisten Fällen eher zeitlich beschränkt und punktuell.

Vermisst wird daher bis heute eine durchgehende Praxis guter Gestaltung unter Einbeziehung der zentralen Faktoren Arbeitsplatz, Arbeitsumgebung, Tätigkeitsgestaltung, Arbeitsorganisation, Arbeitszeitgestaltung, Leistungsbedingungen, Führung und Vereinbarkeit. Der Einfluss der »Tarifverträge Demografie« auf die alternsgerechte Gestaltung von Schichtsystemen oder auf die Vitalisierung betrieblicher Weiterbildung ist ebenso gering geblieben wie ihre Breitenwirkung. Die Demografieverträge und auch die Förderung betrieblicher Demografieprojekte haben in vielen Unternehmen Gestaltungsinseln befördert und gefördert, von einer nachhaltigen Wirksamkeit sind wir allerdings weit entfernt. 
Dagegen erfreuen sich tarifvertragliche Lösungen zur Altersteilzeit einer gleichbleibend hohen Nachfrage und Beliebtheit. Nach wie vor ist der Wunsch der Beschäftigten hoch, vorzeitig in Rente zu gehen. Dies gilt nicht nur für Mitarbeiter*innen in Produktion oder Montage, sondern auch für Beschäftigte in indirekten Tätigkeitssegmenten. Dieser Wunsch ist einerseits eine Antwort auf langjährig erlebte körperliche und psychische Fehlbeanspruchungen. Anderseits resultiert er aus dem Wunsch nach selbstbestimmter Gestaltung des künftigen Lebens, jenseits von betrieblichen Zwängen und profitorientierter Fremdbestimmtheit. Ähnlich lässt sich die hohe Präferenz für mehr freie Tage, anstatt zusätzlichem Entgelt (Regelungen aus der Tarifrunde 2018 zum T-Zug) interpretieren ${ }^{5}$. Der Bedarf an kompensatorischen Maßnahmen aber auch der Wunsch nach mehr Selbstbestimmung über das eigene Leben ist offenkundig vorhanden und bietet auch künftig vielfältige Ansatzpunkte für tarifvertragliche Lösungen, die durch betriebs- und arbeitspolitische Initiativen ergänzt werden müssen.

Anstelle gestaltungsorientierter Vertragsregelungen präferieren Beschäftigte und ihre Interessenvertretungen also in erster Linie Regelungen mit garantierten Gesundheitsschutz- und Altersübergangsleistungen. Neben der Altersteilzeit sind hier zu nennen: erweiterte Pausenregelungen bei getakteter Arbeit oder bei Arbeiten im Schichtbetrieb, Arbeitszeitreduzierung bei langjähriger Schichtarbeit, Entgeltsicherung bei eingeschränkter Leistungsfähigkeit, ein erweiterter Kündigungsschutz für ältere Arbeitnehmer*innen mit entsprechender Beschäftigungsdauer im Unternehmen, mehr Zeitsouveränität, um eigene berufliche Interessen und private Lebenslagen besser miteinander verbinden zu können oder garantierte Ansprüche auf berufliche Weiterbildung. Diese eher regulativ angelegten Maßnahmen lassen sich leichter umsetzen, da sie als verbriefte Individualrechte genutzt werden können.

Eine ähnliche Situation lässt sich für die Regelungen im Arbeits-und Gesundheitsschutz bilanzieren. Der Demografiediskurs hat auf der gesellschaftlichen Ebene zweifelsohne zu Fortschritten geführt. Die Klarstellungen zur Gefährdungsbeurteilung nach dem Arbeitsschutzgesetz und die erneuerte Betriebssicherheitsverordnung zielen in ihren Gestaltungsanforderungen darauf $a b$, eine ganzheitliche Bewertung der Arbeitsbedingungen vorzunehmen und darauf aufbauend, negative Folgen für Gesundheit, Lernförderlichkeit und Persönlichkeitsentwicklung der Beschäftigten abzumildern und durch eine ressourcenorientierte Arbeitsgestaltung, Chancen für die Entwicklung im Erwerbsverlauf zu generieren. Dabei wird auf die Gestaltung optimierter Bedingungen für ein gesundes und kompetentes Älterwerden in Beschäftigung abgehoben. Zugleich besteht die Ver- 
pflichtung, auch die Möglichkeiten und Einschränkungen spezifischer Personen- und Tätigkeitsgruppen zu berücksichtigen. Eine alter(n)sgerechte Arbeitsgestaltung wird explizit als Ziel genannt.

Aber auch hier erkennen wir ein erhebliches Umsetzungs- und Kontrolldefizit. Verschiedene Untersuchungen gehen davon aus, dass deutlich weniger als die Hälfte der Unternehmen eine den Kriterien des Arbeitsschutzgesetzes entsprechende Gefährdungsbeurteilung durchführen. Der Anteil der Unternehmen wird noch geringer, wenn man den Anspruch erhebt, dass körperliche und psychische Faktoren einzubeziehen sind und die Gefährdungsbeurteilung den vollständigen Handlungszyklus - Analyse, Maßnahmenentwicklung, Umsetzung von Maßnahmen, Wirkungskontrollen und ggf. Optimierung der durchgeführten Maßnahmen - beinhalten muss und in regelmäßigen Abständen oder anlassbezogen zu wiederholen ist.

Auch die Praxis bei der Umsetzung der Betriebssicherheitsverordnung fällt weit hinter ihrem Regelungserfordernis zurück. Sie regelt, dass vor Inbetriebnahme neuer Technologien, Maschinen, Hilfsmittel oder grundlegender arbeitsorganisatorischer Veränderungen mögliche künftige körperliche oder psychische Fehlbeanspruchungen durch eine vorausschauende Gefährdungsbeurteilung identifiziert werden müssen. Kritische Punkte gilt es im Beschaffungsprozess zu berücksichtigen oder durch geeignete Maßnahmen im Implementierungsprozess abzumildern. Die Betriebssicherheitsverordnung eröffnet einen Weg zu einer vorausschauenden Gestaltung alternsgerechter Arbeitsbedingungen. Es ist schmerzhaft, dass sie in den allermeisten Betrieben nur rudimentär, d.h. verkürzt auf Sicherheitsaspekte oder auf körperliche Fehlbeanspruchungen angewendet wird. Eine vollständige Betrachtung künftiger Arbeitsbedingungen, also der Einschluss der Bedingungen des Arbeitsplatzes, der Arbeitsumgebung, der Tätigkeitsgestaltung, der Arbeitszeitgestaltung, der Arbeitsorganisation und der Leistungsbedingungen als einzelne Faktoren und ihren spezifischen Wechselwirkungen findet in der Regel nicht statt.

Es zeigt sich, dass die Umsetzung von gesetzlichen Bestimmungen ebenso einer wirksamen Kontrolle bedarf, wie dies bei den Tarifverträgen der Fall ist. Diese Kontrolle muss regelmäßig erfolgen und folgende Aspekte beinhalten: Erstens: Werden diese Regelegungen angewendet? Zweitens: Werden die Regelungen entsprechend den Zielsetzungen angewendet? Drittens: Werden mit der Anwendung der Regelungen die gewünschten Effekte erzielt? Dies gilt besonders bei Prozessvereinbarungen. 


\section{Wie erklärt sich die fehlende Durchsetzungskraft?}

\section{Handlungsbedarf nicht sofort und überall vorhanden}

Alternde Belegschaften sind nicht per se ein Problem und Demografieverträge verwandeln sich nicht per se in eine Problemlösung, solange die betrieblichen Leistungsanforderungen trotz fortschreitender Alterung der Belegschaften von den Beschäftigten und den betrieblichen Entscheidungsträgern ohne generationenspezifische Unterstützungsmaßnahmen zu bewältigen sind. M.a.W.: Demografie- oder auch andere Tarifverträge, die der Ausgestaltung von industriellen Beziehungen dienen, erfüllen erst dann ihren Zweck, wenn ein besonderer Regelungsbedarf vorhanden ist und die Vertragsparteien daraus wechselseitigen Nutzen ziehen können.

In vielen Unternehmen fehlte während der 2000er-Jahre nicht das Problembewusstsein für die möglichen demografiebedingten Personalengpässe und Produktivitätsrisiken. Es mangelte aber hauptsächlich an konkreten Gründen, initiativ zu werden. Die Mehrheit der Unternehmen sah sich vor der Finanzkrise noch nicht als vom Altersstrukturwandel betroffen. Dieser Eindruck konnte leicht entstehen und sich verfestigen, denn die Belegschaften geben in ihrer Alterszusammensetzung grundsätzlich kein einheitliches Bild ab. Sie sind von regionalen Differenzen geprägt und variieren stark nach Bildungsniveau, Unternehmensgröße sowie dem Migrationshintergrund der Beschäftigten. Der Handlungsdruck in der Mehrheit der Belegschaften entwickelte und erhöhte sich erst nach und nach. Er wurde vor allem seit 2012 mit der Anhebung des Renteneintrittsalters auf 67 Jahre spürbarer. So erhöhte sich die Erwerbsquote der Altersgruppe 55 bis unter 60 Jahre im Zeitraum von 2008 bis 2018 von 75,2 Prozent auf 83 Prozent. Die Erwerbsquote der 60- bis 64-Jährigen legte im gleichen Zeitraum am meisten zu - von 37,8 Prozent auf 62,5 Prozent. In der Altersgruppe 65 Jahre und älter stieg der Erwerbsanteil nahezu um das Doppelte - von 3,9 Prozent auf 7,5 Prozent (Bundesagentur für Arbeit 2019). Und dennoch unterblieben strukturelle Investitionen in eine alterns- und altersgerechte Arbeitsgestaltung und Personalpolitik, weil die Unternehmen an Konzept und Strategie des kontrollierten Beschäftigungsabbaus durch vorzeitigen Altersübergang festhalten konnten und solche Spielräume offensiv nutzten. 


\section{Widersprüche und Problemdruck fübrten zu betrieblichen Einzelprojekten}

Die Initiativen von Betriebsräten und Gewerkschaften für eine alter(n)sgerechte Arbeitsgestaltung sind davon abhängig, Konstellationen, Entwicklungen und Trends zu identifizieren, mit denen eine Anschlussfähigkeit zu den Interessen des Managements hergestellt werden kann. Betriebliche Initiativen und Gestaltungsprojekte für eine alter(n)sgerechte Arbeitsgestaltung setzten dabei an vier zentralen Widersprüchen der betrieblichen Arbeitspolitik an:

Erstens: Mit zunehmender Tätigkeitsdauer in belastenden Arbeitsbereichen, insbesondere im Bereich der Produktion und der Montage steigen die Krankenquoten stark an. Die betriebliche Einsatzfähigkeit wird aus gesundheitlichen Gründen eingeschränkt. Hieraus ergeben sich erhebliche Mehrkosten aber auch arbeitsorganisatorische Probleme im Personaleinsatz (Anlauft 2017). Arbeitsbedingte gesundheitliche Restriktionen und betriebliche Leistungsfähigkeit geraten in Widerspruch.

Zweitens: Tätigkeiten mit geringem Arbeitsumfang, die sich ständig wiederholen und in ihrer Ausführung genau vorgeschrieben sind, untergraben die geistigen Fähigkeiten der Mitarbeiter*innen auf diesen Arbeitsplätzen. Der beschleunigte technologische und arbeitsorganisatorische Wandel (Arbeit 4.0) führt aber zu neuen fachlichen Anforderungen. Arbeitsbedingte Einschränkungen der Lernfähigkeit und neue Anforderungen geraten in Widerspruch.

Drittens: Eine nachhaltig wirksame Bewältigung des Wandels ist ohne das aktive Handeln der Beschäftigten nicht zu erreichen. Diese Bereitschaft wird aber durch die bisherige Praxis der Arbeitsgestaltung systematisch untergraben. Arbeitsbedingte Motivationsverluste und Anforderungen für ein nachhaltig wirksames Veränderungsmanagement geraten in Widerspruch.

Viertens: Die veränderte Arbeitsmarktsituation führt - abhängig von Regionen und Tätigkeitsgruppen - zu größeren Schwierigkeiten, zeitnah geeignete Arbeitnehmerinnen zu rekrutieren. Hohe Übergänge in Rente, insbesondere bei Beschäftigten mit ausgeprägten erfahrungsgebundenen Fach- und Spezialkompetenzen, erhöhten den Druck auf das Personalmanagement, durch geeignete Maßnahmen der Personalrekrutierung, Personalbindung, der Personalentwicklung, des Potenzialerhalts und des Knowhow Transfers diesem Umstand Rechnung zu tragen (Anlauft 2018). 


\section{Gestaltungszäsur Finanzmarktkrise}

$\mathrm{Zu}$ allem Übel ereignete sich auch noch mitten in der Anlaufphase des Demografievertrages in der Eisen- und Stahlindustrie die Finanzmarktkrise, die in der Stahlindustrie 2009 für einen Einbruch von durchschnittlich $30 \%$ der Jahreswirtschaftsleitung sorgte. Die Sicherung von Beschäftigung und der Standorte sowie die Ankurbelung der Wirtschaft (»Abwrackprämie«) standen naturgemäß im Vordergrund. Das Demografieprojekt erhielt einen nachhaltigen Dämpfer.

\section{Komplexität und Prozesscharakter der Tarifregelung}

Ein weiteres Erklärungsmuster, weshalb den Demografieverträgen die Durchsetzungskraft für eine andere Ausrichtung der Personalpolitik fehlte, nimmt die Komplexität und den Prozesscharakter der Tarifregelung selbst in den Blick. Kapitza u.a. verweisen diesbezüglich auf einen erhöhten Beratungs- und Unterstützungsbedarf der betrieblichen Umsetzungsakteure. Bei der Umsetzung von Prozessvereinbarungen à la Demografievertrag Stahl braucht man einen langen Atem. Analyse, Maßnahmenentwicklung, Gestaltungsarbeit und Wirksamkeitskontrolle sind eingebunden in einen Prozess der kontinuierlichen Verbesserung betrieblicher Initiativen. Es handelt sich um eine Daueraufgabe und nicht um ein zeitlich befristetes Projekt. Das Vorgehen muss für unterschiedliche »Wetterlagen" (Krise, Wachstum, verschiedene Reorganisationsmuster) tauglich gestaltet sein. Alter(n)sgerechte Arbeitsgestaltung zielt auf eine langfristige Gestaltung und eignet sich nicht für kurzfristige Kostenziele. Es geht um Früherkennung und weniger um die Behandlung von Spätfolgen (Stork/Widuckel 2018). Die Anforderungen aufgrund des breiten Spektrums möglicher Regelungsgegenstände, die Notwendigkeit, freiwillige teilmitbestimmte Betriebsvereinbarungen abzuschließen und besondere fachliche Anforderungen aus dem Prozessmonitoring erweisen sich tendenziell als überfordernde und umsetzungshemmende Faktoren. Andere Autoren (Bellmann u.a. 2018; Latniak u.a. 2010; Morschhäuser 2006) erkennen Handhabungsdefizite von Analysetools (Altersstrukturanalyse, Qualifikationsbedarfsanalyse, Gefährdungsbeurteilung) als Ursachen dafür, dass die vorgeschriebene Datenermittlung kaum in Maßnahmenpakete mündete. 


\section{Kostenoptimierung versus alter $(n)$ sgerechter Arbeitsgestaltung}

Viele Demografieprojekte stehen und standen in Widerspruch zu anderen betrieblichen Veränderungsprojekten. Nach der arbeitspolitischen LeanPhase der 1990er-Jahre breiteten sich im darauffolgenden Jahrzehnt - in der Hochphase des Shareholder-Kapitalismus - sogenannte ganzheitliche Produktionssysteme (GPS) wellenförmig über den engen Bereich der Automobilindustrie hinaus aus, sei es isoliert als Einzelmaßnahme (z.B. als 5 S-Ressourceneffizienzprogramm) oder viel seltener als unternehmensweites durchgängiges Shopfloor-Management-Konzept (Seibold u.a. 2016). Montageprozesse und andere Tätigkeitsumfänge, die der GPS-Logik folgend von indirekten Tätigkeitsanteilen befreit und kurzzyklischer organisiert wurden, führten jedoch im Allgemeinen zur Leistungsintensivierung und Dequalifizierung der Beschäftigten (Hirsch-Kreinsen und Weyer 2013). Eine Standardisierung der Arbeitsprozesse, vermehrtes Arbeiten im Zeittakt und die Reduzierung von Pufferzeiten erhöhten den Anteil einseitiger Belastungen. Eine bessere ergonomische Gestaltung von Bedingungen am Arbeitsplatz und der Arbeitsumgebung, die ebenfalls mit der Einführung des GPS vorgenommen wurden, konnten diese negativen Wirkungen für ein gesundes und kompetentes Älterwerden in Beschäftigung nicht kompensieren.

Zugespitzt formuliert: Demografieprojekte konnten sich nicht als Standardgestaltungslösungen etablieren, weil sie nicht an die technologische oder arbeitsorganisatorische Optimierung von Arbeitsprozessen angekoppelt waren, die sich ihrerseits vollständig auf eine kurzfristige Kostenoptimierung fokussierte. Alter(n)sgerechte Arbeitsgestaltung und die Gestaltung von ganzheitlichen Produktionssystemen verfolgten unterschiedliche Ziele und wurden als getrennte Vorgänge behandelt. Selbst wenn in den Regelungen der GPS-Vereinbarungen das Ziel einer alter(n)sgerechten Arbeitsgestaltung enthalten war, so fehlten die hinlänglichen Konkretisierungen in der konkreten Ausgestaltung. Alter(n)sgerechte Arbeitsgestaltung wurde nicht selten gleichgesetzt mit verbesserter Ergonomie. Erweiterte Tätigkeitsinhalte und angemessene Leistungsbedingungen wurden vernachlässigt. Sie standen in Konflikt mit den Prinzipien einer »schlanken Produktion «, die auf kurzfristige Kostenoptimierung setzte und dabei die Gesundheits- und Persönlichkeitsinteressen der Beschäftigten vernachlässigte.

Je nach Rationalisierungsgrad und Kostensenkungsziel kam es dabei also in den meisten Fällen zur Verschlechterung der Arbeitsbedingungen. Ansätze innovativer Arbeitspolitik, die das Ziel einer nachhaltigen Verbes- 
serung der Arbeits- und Beschäftigungsbedingungen verfolgten, standen daher vor und nach der globalen Finanzkrise unter erheblichem Druck.

\section{Betriebliche Flexibilisierung versus alter(n)sgerechte Arbeitsgestaltung}

Auftragsschwankungen, kürzere Lieferfristen und geringere Vorratshaltung führten zu einer Flexibilisierung bei den Tages-, Wochen und Jahresarbeitszeiten, der betrieblichen Arbeitsorganisation aber auch zur Just-intime-Ausgestaltung der Arbeitsverhältnisse. Möglich wurde dies durch Betriebsvereinbarungen zu Arbeitszeitkonten und durch neue gesetzliche Regelungen zum erweiterten Einsatz von Leiharbeit ab 1. Januar 20036. Dadurch veränderte sich für die Unternehmen auch der arbeitsmarkt- und personalpolitische Handlungsrahmen ${ }^{7}$. Für die Betriebe war es nun deutlich attraktiver, ihre Bedürfnisse nach flexiblem Arbeitseinsatz über Leiharbeit zu decken. Alterssensible und lernförderliche Personalkonzepte mussten sich ab diesem Zeitpunkt gegen ein weiteres Gegenkonzept behaupten.

Mit der intensiven Nutzung atypischer Beschäftigungsformen konnten die Unternehmen eine Beschäftigungsstrategie verfolgen, die sich als kontraproduktiv für gesundheits- und lernförderliche Arbeitsgestaltung erwies. Besonders für Intensivnutzer von Leiharbeit im verarbeitenden Gewerbe, die eine Lohnsenkungsstrategie verfolgten, machten Investitionen in innovative Arbeitsgestaltung keinen Sinn (Dunckel 2014). Das Personalkonzept des temporären Zukaufs von überlassenen Kompetenzen war und ist zudem eine Einladung, die Personalentwicklung im Unternehmen zu vernachlässigen (Bellmann/Kühl 2007) und Stammbeschäftigte, die in Rente gehen, nicht mehr dauerhaft zu ersetzen.

Begünstigt wurde diese Entwicklung zudem durch eine hohe Arbeitslosigkeit zu Beginn der 2000er-Jahre. Dieser Umstand beförderte eine Personalbemessung, die sich eher am Minimum des Auftragsvolumens orientierte. Lag das Auftragsvolumen darüber, wurden die erforderlichen Ressourcen durch Ausdehnung von Mehrarbeit (Arbeitszeitkonten), Erhöhung der Anzahl der Leiharbeitnehmer*innen oder Ausdehnung von Werkverträgen mobilisiert. Diese eher kurzfristige Reaktion auf eine schwankende Nachfragesituation ersetzte zunehmend eine mittelfristige Personalplanung und begünstigte eine erhebliche Leistungsverdichtung in den Unternehmen. Mit dieser Politik der "permanenten Überlastung" der Arbeitsbereiche wurden Ziele einer alter(n)sgerechten Arbeitsgestaltung unterlaufen. 


\section{Perspektiven und Spielräume einer integrierten Personal- und Arbeitspolitik}

\subsection{Demografiesensible Personalarbeit bleibt auf der Tagesordnung}

Der Generationenwechsel in den Unternehmen ist noch lange nicht abgeschlossen. Die Betriebe der M\&E-Industrie und der weiteren Branchen werden bis in die 30er-Jahre mit einem relevanten Anteil älterer Beschäftigter konfrontiert sein. Für sie und die jüngeren Nachwuchskräfte ist das Niveau körperlicher und psychischer Belastungen bislang nicht geringer geworden. Die (Selbst-)Zweifel an der Fähigkeit vieler Arbeitnehmer"innen, die Arbeitsfähigkeit bis zum regulären Renteneintrittsalter unbeschadet zu erhalten, sind nach wie vor hoch. Die Reaktionen der Unternehmen auf diese Situation sind unterschiedlich. Betriebliche Strategien variieren zwischen der Externalisierung der Folgen langjährig schlechter Arbeitsgestaltung (Altersteilzeit, Verlagerung schlechter Arbeitsbedingungen auf Leiharbeitnehmer*innen) und Strategien des »Augen zu und durch«. In der zweiten Phase der Coronakrise mehren sich zudem die Anzeichen für gezielte Anpassungen der Personalkapazitäten bei Älteren. Wir finden verstärkte Anstrengungen bei der Nachwuchsrekrutierung ebenso wie eine Arbeitsgestaltung, die ein gesundes und kompetentes Älterwerden ermöglichen.

Das Ausmaß des Fachkräftebedarfes in den Unternehmen ist uneinheitlich und führt nicht zwangsläufig dazu, dass sich das Personalmanagement intensiv mit der Sicherung von Fachpersonal beschäftigt. Unternehmen mit stabilem Beschäftigungsniveau treibt dagegen stärker die Frage um, wie durch eine systematische Nachfolgeplanung und durch die Weiterentwicklung der Systeme der betrieblichen Weiterbildung der Verlust an Fachqualifikationen ersetzt bzw. kompensiert werden kann. Nicht selten findet aus Sicht des Managements auch eine differenzierte Betrachtung des Potenzials älterer Arbeitnehmer*innen statt. Zugespitzt formuliert werden Beschäftigte in mittleren und höher qualifizierten Tätigkeiten eher als Potenzial mit erweitertem Erfahrungswissen betrachtet, während Beschäftigte in Tätigkeiten mit geringerem Tätigkeitsumfang mit zunehmendem Alter als Kostenfaktor wahrgenommen werden. Welche Lösungen sich im Einzelnen durchsetzen und ob in der jeweiligen betrieblichen Gemengelage daraus eine kohärente Gesamtstrategie entsteht, hängt ab von den spezifischen Rahmenbedingungen des Arbeitsmarktes (Fachkräfteengpässe oder überzählige Arbeitskräfte) und nicht zuletzt von der Stärke und der Gestaltungskompetenz der betrieblichen Interessenvertretung. 


\subsection{Strategische Personalplanung als neue Klammer}

Der aktuelle Handlungsdruck im Bereich der Metall- und Elektroindustrie, Ressourcen in eine alternsgerechte Arbeitsgestaltung zu investieren, ist erheblich und wird in der nächsten Dekade voraussichtlich noch an Dynamik zulegen. Die zentrale arbeitspolitische Aufmerksamkeit gilt jedoch den neuen Geschäftsmodellen der Plattformökonomie und den digitalisierungsgetriebenen technologischen und arbeitsorganisatorischen Veränderungen. Initiativen für eine alter(n)sgerechte Arbeitsgestaltung werden dadurch in den Hintergrund gedrängt, dürften jedoch ihre Chance bekommen, wenn sie sich wegen der schieren Anzahl älterer Beschäftigter in diesem Kontext betrieblicher Transformation verorten, lautstark zu Wort melden und eine strategische Personalplanung (INQA, 2018) die verschiedenen betrieblichen Entwicklungslinien und Gestaltungsaufgaben miteinander verbindet. Aktivitäten für eine alter(n)sgerechte Arbeitsgestaltung müssen raus aus der Nische eines Ein-Punkt-Projektes. Sie werden nur als integrierter Bestandteil betrieblicher Arbeitspolitik wirksam werden. Die Verbindung mit einer strategischen Personalplanung bietet die Chance, den Handlungsrahmen zu erweitern und das Thema vom Rand ins Zentrum zu manövrieren.

Aus dem Blickwinkel von Betriebsräten sind an dieser Stelle alle klassischen Arbeitsfelder der Arbeit der Interessenvertretung angesprochen. Sie sind gefordert, ihrer traditionelle Schutz- und Gestaltungsfunktion für ältere und älterwerdende Beschäftigte zu aktivieren. Sie sollten einerseits mit Forderungen nach Beschäftigungssicherung und Arbeitsgestaltung intervenieren. Zum andern gilt es, eine systematische Personalentwicklung anzustoßen, welche die Ergebnisse einer quantitativen Personalplanung (Geschäftsentwicklung, Nachfolgeplanung, Personalbemessung) und qualitative Veränderungen durch neue Tätigkeitszuschnitte, Aufgaben und Technologien berücksichtigt.

\subsection{Demografiesensible Personalarbeit als Strategieziel}

Eine alter(n)sgerechte Arbeitsgestaltung als betriebliches Handlungsfeld hängt also entscheidend von der künftigen Ausgestaltung der betrieblichen Arbeitspolitik ab. Diese wiederum wird sich an den zentralen Linien betrieblicher Entwicklungen und ihrer Reorganisationsprozesse ausbilden. Dabei erscheint es aus heutiger Sicht vollkommen offen, welchen Platz Unternehmen und Interessenvertretungen dem betrieblichen Alternsmanagement einräumen. Die Ziele einer alter(n)sgerechten Arbeitsgestaltung 
bleiben zwar aktuell, sie müssen aber mit den zentralen Zukunftsthemen der Unternehmen und der Beschäftigten verschmolzen werden. Alter $(\mathrm{n})$ sgerechte Arbeitsgestaltung ist daher ein wichtiges Ziel im Rahmen einer größeren Handlungsstrategie gewerkschaftlicher Betriebspolitik und der Einflussnahme für eine menschengerechte Gestaltung von Arbeit. Offen ist, ob es den Interessenvertretungen gelingen wird, Beschäftigungssicherung und menschengerechte Arbeitsgestaltung auch im Sinne einer demografiesensiblen Personalarbeit einzufordern und mitzugestalten. Dafür sprechen die Gestaltungsrenditen einer high-road Strategie in der Unternehmensentwicklung mit adäquaten Maßnahmen einer innovationsförderlichen Arbeitsgestaltung. Dagegen sprechen Konstellationen mit möglichen Abwehrkämpfen nach dem Prinzip »Hauptsache Arbeit, egal wie schlecht sie auch immer sein mag«.

\subsection{Gestaltungsprozesse frühzeitig beeinflussen}

Schon heute zeichnet sich ab, dass technologische und arbeitsorganisatorische Veränderungen, die mit einer Arbeitswelt 4.0 verbunden sind, sehr unterschiedliche Wirkungen für ein gesundes und kompetentes Älterwerden in Beschäftigung mit sich bringen können. Digitale Assistenzsysteme in der Industrie können z.B. die Handlungsspielräume in der Produktion, Montage und im Büro erhöhen, Arbeit aufwerten und zu deutlich erweiterten Qualifikationsanforderungen mit Perspektiven für die eigene berufliche Entwicklung führen. Digitale Assistenzsysteme können aber auch die Handlungsspielräume einengen, den Mensch zum Befehlsempfänger der Maschine machen, einseitige Belastungen in der Arbeitsausführung erhöhen, die Qualifikationsanforderungen reduzieren und die Leistungserbringung der Beschäftigten lückenlos überwachen. Zentral sind hierbei die arbeitspolitischen Gestaltungskonzepte. An der Ausrichtung und Qualität dieser Gestaltungslösungen entscheidet sich, ob die Beschäftigung in der Arbeitswelt 4.0 auch für ältere Arbeitnehmer*innen, die langjährig in Tätigkeiten mit einem hohen Ausmaß körperlicher und psychischer Fehlbeanspruchungen tätig waren, geeignet ist und mit diesen Bedingungen ein arbeitsbedingtes Voraltern von Arbeitnehmer*innen jüngeren und mittleren Alters verhindert werden kann. Die Einflussnahme auf diese Gestaltungsprozesse muss - wie bei allen komplexen technologischen Systemen - sehr frühzeitig, d.h. bereits im Planungsprozess erfolgen und durch einen Einführungsprozess unter Beteiligung der Beschäftigten vorgenommen werden. 


\subsection{Zukunftsvereinbarungen}

"Tarifverträge Demografie« in der bekannten Form besitzen aus unserer Sicht nicht die Akzeptanz und das notwendige Potenzial, solche tiefgreifenden Gestaltungsprozesse anzustoßen und ihnen ihren Stempel aufzudrücken. Gegenwärtig erscheint der Abschluss von Transformations- oder Zukunftsvereinbarungen als ein geeigneteres Mittel, um festzulegen, wie ein Interessenausgleich bei der gemeinsamen Gestaltung der Digitalisierung erreicht werden kann. Alter(n)sgerechte Arbeitsgestaltung sollte dabei zu den prioritären Vereinbarungselementen zählen, damit die Entwicklungs- und Gesundheitsinteressen der Beschäftigten in den betrieblichen Transformationsprozessen zur Geltung kommen.

Der gegenwärtige betriebliche Strukturwandel, der die Arbeits- und Beschäftigungsbedingungen deutlich verändert und die Zukunftsperspektiven einzelner Beschäftigtengruppen zum Teil grundlegend infrage stellt, besteht aus mehreren und gleichzeitigen Transformationsvorgängen: Neue Geschäftsmodelle, Digitalisierung, Altersstrukturwandel, Dekarbonisierung von Produkten und Geschäftsmodellen überschneiden und überlagern sich. Die Rationalisierungs- und Anpassungsdynamik der digitalen Transformation trifft demnach in Unternehmen und Organisationen auf älter werdende Belegschaften und verlangt von ihnen die Bereitschaft zur Anpassung ihrer Qualifikation, ihrer Einsatzflexibilität und ihres Beteiligungs- und Vorgesetztenverhaltens (z.B. in agilen Arbeitsformen). Die Digitalisierung vernetzt Beschäftigte und ihre (Home Office-)Arbeitsplätze in allen Altersgruppen weit stärker als bisher und dehnt die jetzt schon vorhandenen Risiken entgrenzter Arbeit möglicherweise noch weiter aus. Die Folgen des Strukturwandels für die Ausgestaltung von Arbeit, für die $\mathrm{Zu}-$ sammenarbeit und Kommunikation und für die berufliche Entwicklung nicht nur bildungsferner Beschäftigtengruppen sind tiefgreifend. Gleichwohl ist zu betonen: trotz des Einsatzes fortgeschrittener Technologie sind das Erfahrungswissen und gut qualifizierte Beschäftigte das unentbehrliche Fundament reibungsloser Produktions-, Entwicklungs- und Verwaltungsabläufe.

Die Corona-Pandemie hat zudem gezeigt, dass Arbeits- und Gesundheitsschutz kein Add-on sind. Vielmehr ist die Sicherstellung von Arbeitsund Gesundheitsschutz durch Kommunen und Länder Voraussetzung und Basis angepasster (branchen-)tarifvertraglicher Vereinbarungen der Sozialpartner. Die Alterung der Erwerbsbevölkerung wirft die Frage auf, ob die Anpassung der Arbeit an den Menschen nicht alleine den Sozialpartnern überlassen werden kann oder ob Sozialversicherungen und Behörden viel 
stärkere Impulse zur Verwirklichung alters- und alternsgerechter Arbeitsbedingungen geben müssen.

Neue tarifvertragliche Zukunftsvereinbarungen - so unser Plädoyer können zu einer verbesserten arbeitspolitischen Praxis aus Sicht der Beschäftigten führen. Dabei müssen beschäftigungs- und arbeitspolitische Schutzbedürfnisse und Schutzstandards aufgegriffen, aber auch den entwicklungsorientierten Arbeitsinteressen der Beschäftigten Rechnung getragen werden. Die einzelnen Elemente dieser Tarifverträge müssen hinreichend konkrete Ziele und Verfahren für das gemeinsame Handeln von Management und Interessenvertretung festlegen, aber auch belastbare Rechtsansprüche für die Beschäftigten enthalten. Das betriebliche Handeln muss durch überbetriebliche Lernprozesse und einen erleichterten Ressourcenzugang für die betriebliche Interessenvertretung unterstützt werden. Die Umsetzungspraxis muss überprüft werden. Dies beinhaltet die Überprüfung nach Vollständigkeit, Qualität und in Hinblick die konkreten Wirkungen der Tarifverträge. Diese Elemente unterstützen eine erweiterte und verbesserte Praxis der Umsetzung, des Lernens und der verbesserten Wirkungen im Interesse der Beschäftigten. Dadurch kann eine neue Dualität von Tarifverträgen und betrieblichen Regelungen entstehen. Deutlich erweiterte tarifvertragliche Standards werden durch betriebliche Regelungen konkretisiert und die Handlungsoptionen durch regionale oder branchenbezogene Unterstützungsstrukturen erweitert.

Aus den Erfahrungen mit »Tarifverträgen Demografie « lassen sich so für den Abschluss von »Tarifverträgen Transformation« folgende Essentials ableiten:

1. Inhaltlich geht es um Vereinbarungen zur betrieblichen Innovationsund Investitionspolitik (Geschäftsmodelle, Produkte, Technologien) und ihre Wirkungen für die betriebliche Arbeitspolitik. Vereinbarungsbedürftig sind damit insbesondere die Themen Standort- und Beschäftigungssicherung, interner Arbeitsmarkt, Arbeitsgestaltung, Gesundheit, Arbeitszeit, Inklusion, Leistung und Kontrolle, Qualifizierung.

Dabei müssen Eckpunkte und Zielgrößen zu

- den Gestaltungszielen (definierte Veränderungsprojekte),

- den eingesetzten Ressourcen (Investitionsfonds, definierte Investitionen/Budgets),

- den Abläufen (gemeinsame Arbeits- oder Projektgruppen von Arbeitgeber"in und Betriebsrat) und

- den Entscheidungsmechanismen (paritätisch besetzter Steuerkreis) festgelegt werden. 
2. Die Individualrechte der Beschäftigten müssen gestärkt werden: Zugang zu Weiterbildung, Freistellungsmöglichkeiten entsprechend den beruflichen Entwicklungsvorstellungen und privaten Bedingungen, gestärkte Initiativrechte für gesundes und kompetentes Arbeiten, definierte Mitwirkungsrechte bei Einführung neuer Technologien und arbeitsorganisatorischer Veränderungen.

3. Wesentlich sind erweiterte Informations-, Initiativ- und Mitbestimmungsrechte für Betriebsräte bei allen Fragen der strategischen Personalplanung (quantitativ und qualitativ). Diese zeitlich umfangreicheren und fachlich komplexer werdenden Anforderungen an die Arbeit der betrieblichen Interessenvertretung bedürfen erhöhter Freistellungsressourcen. Ferner muss der Zugang zu externer, fachlich spezialisierter Beratung erleichtert werden.

4. Die Unternehmen müssen mit Sanktionen rechnen, wenn Umsetzungsdefizite im Bereich des Arbeits- und Gesundheitsschutzes und Regelverstöße gegen diese Vereinbarungen festgestellt werden.

5. Auf der Branchenebene werden paritätisch besetzte "Agenturen für gute Transformation" installiert, die - finanziert aus einem Tariffonds bzw. einem Mindestanteil der Lohn- und Gehaltssumme - Überwachungs-, Entwicklungs- und Kommunikationsaufgaben übernehmen.

- Die Agenturen überwachen Regelungen aus diesen Vereinbarungen, belohnen regelkonformes Verhalten, sanktionieren regelwidriges Verhalten und berichten jährlich zum Umsetzungsstand in den Unternehmen.

- Sie fördern von zentraler Stelle aus gute Transformationspraxis, indem sie Modellprojekte initiieren, Gestaltungshilfen erstellen, Diskussions- und Lernprozesse anregen, Forschungsthemen identifizieren und entsprechende Vorhaben initiieren. Des Weiteren sind sie ein Forum für den regionalen industriepolitischen Diskurs zur Innovationspolitik und zur Zukunft der Arbeit.

\subsection{Forschungsbedarf zur Mehrfach-Transformation}

Die beschriebenen multiplen Veränderungsprozesse, die in den Betrieben an verschiedenen Stellen zu lokalisieren sind, lösen zwangsläufig neue Forschungsfragen aus, ältere Forschungsfragen stellen sich vor diesem Hintergrund neu. Was es zu beforschen gäbe, wäre der Zusammenhang von digitaler Transformation und demografischen Veränderungen mit dem Ziel, in kurzer Zeit mehr Handlungs- und Gestaltungssicherheiten für eine zukunftsfeste Weiterbildung und für neue Impulse bei der Gestaltung lern- 
förderlicher und gesundheitsverträglicher Arbeit zu gewinnen. Erwünscht sind auch Detailerkenntnisse zur Subjektseite technologischer Veränderungen: Wie können Transformationsprozesse angestoßen werden, dass sie den unterschiedlichen Bedürfnissen und Kompetenzen der Generationen und Berufsgruppen gerecht werden? Worauf haben die verantwortlichen Prozessowner zu achten, damit der Fachlichkeit, dem Entdeckergeist, dem Produzentenstolz und den Lebensleistungen der einzelnen Beschäftigten gebührend Rechnung getragen wird.

Es bedarf fundierter Analysen, die sowohl bestehende Defizite wie auch die Entwicklungschancen und -risiken der Transformationsvorgänge kenntlich machen. Es ist notwendig, mehr zu erfahren über Widersprüche, Konfliktpotenziale und systemische Wechselwirkungen zwischen unterschiedlichen Dimensionen des Wandels. Systematisch zu durchleuchten wäre ferner das Wirkungsverhalten von Vereinbarungsinstrumenten und Personalstrategien.

\section{Literatur}

Anlauft, W. (2017): Gestaltung von Job Rotation in der Produktion. In: Richter, G., Hecker, C., Hinz, A. (Hrsg.): Produktionsarbeit in Deutschland - mit alternden Belegschaften, Erich Schmidt Verlag, Berlin.

Anlauft, W. (2018): Zukunftsfähiges Kompetenzmanagement - Prospektiv, lebensphasenorientiert und regional flankiert, Eigenverlag, Nürnberg.

Bellmann, L., Brandl, S., Dummert, S., Guggemos, P., Leber, U., Matuschek, I. (2018): Altern im Betrieb. Alterung und Alter(n)smanagement in kleineren und mittleren Unternehmen - vom Einzelfall zur professionalisierten Systematik, Study Nr. 393, Düsseldorf.

Bellmann, L., Kühl, A. (2007): Weitere Expansion der Leiharbeit? Eine Bestandsaufnahme auf Basis des IAB-Betriebspanels, Studie im Auftrag der Hans-BöcklerStiftung.

Bertelsmann Stiftung (2013): Alternde Belegschaften - Chancen, Risiken und Herausforderungen. Impulse 2013/02.

BIBB/BauA (2019): Grundauswertung der BIBB/BAuA Erwerbstätigenbefragung 2018 - Vergleich zu Grundauswertungen 2006 und 2012, Dortmund, Berlin, Dresden.

Bundesagentur für Arbeit (2020): Aktuelle Entwicklungen in der Zeitarbeit. Berichte: Blickpunkt Arbeitsmarkt, Nürnberg.

Bundesagentur für Arbeit (2019): Situation von Älteren. Berichte: Blickpunkt Arbeitsmarkt, Nürnberg. 
Dunckel, H. (2014): Zukunftsorientierte Arbeitsgestaltung. In: Badura, B., Ducki, A., Schröder, H., Klose, J., Meyer, M. (Hrsg.): Fehlzeiten-Report 2014: Erfolgreiche Unternehmen von morgen - gesunde Zukunft heute gestalten, S. 187-194.

Fröhler, N., Fehmel, T. (2018): Tarifvertragliche Regulierung sozialer Sicherung: Deutschland und Österreich im Vergleich, Working Paper Forschungsförderung 076, Hans-Böckler-Stiftung, Düsseldorf.

Hirsch-Kreinsen H., Weyer, J. (Hrsg.) (2013): Wandel von Industriearbeit. Herausforderungen und Folgen neuer Produktionssysteme in der Industrie, Soziologisches Arbeitspapier Nr. 32/2013.

Kapitza, I., Kopp, D., Metzmann, E., Schlegel, U., Schneider, C. (2013): Projektabschlußbericht Umsetzung von Demografie-Tarifverträge. In: IG BCE, QFC $\mathrm{GmbH}$ (Hrsg.), Hannover.

INQA (2018): Strategische Personalplanung leicht gemacht, Berlin.

Katenkamp, O., Georg, A., Martens, H. (2012): Gestaltung des demografischen Wandels durch Tarifvertrag? In: WSI-Mitteilungen 6/2012, S. 427-434.

Klippert, J. (2014): Alter(n)skritische Merkmale von Tätigkeiten, unveröffentlicher Vortrag, Frankfurt.

Latniak, E., Voss-Dahm, D., Elsholz, U., Gottwald, M., Gerisch, S. (2010): Umsetzung demografiefester Personalpolitik in der Chemischen Industrie. Inhaltliche und prozessuale Analyse betrieblicher Vorgehensweisen, Abschlussbericht für die Hans-Böckler-Stiftung, Düsseldorf.

Morschhäuser, M. (2006): Reife Leistung. Personal- und Qualifizierungspolitik für die künftige Altersstruktur, Forschung aus der Hans-Böckler-Stiftung, Bd. 72, Berlin.

Prognos AG (2010): Recherche und Darstellung betrieblicher Demographieprojekte. Endbericht an die Bundesanstalt für Arbeitsschutz und Arbeitsmedizin (BAuA), Berlin.

Seibold, B., Schwarz-Kocher, M., Salm, R. (2016): Ganzheitliche Produktionssysteme (GPS). Hans-Böckler-Study Nr. 340, Düsseldorf 11/2016.

Stork J., Widuckel W. (2018): Betriebliches Alter(n)smanagement - sechs Spannungsfelder betrieblicher Praxis. In: WSI Mitteilungen 1/2018 Schwerpunktheft, Nomos Verlag Baden-Baden. 
Tullius, K., Freidank, J., Grabbe, J., Kädtler, J., Schroeder, W. (2012): Perspektiven alter(n)sgerechter Betriebs- und Tarifpolitik. In: WSI-Mitteilungen 2/2012, S. 113-123.

Volkholz, V. (1993): Die demografische Revolution erfasst auch die Betriebe, Frankfurter Rundschau v. 22.11.1993.

\section{Referenzen}

1 Die Politik der Destabilisierung und Vermarktlichung der Alterssicherung erfolgte über eine Vielzahl von gesetzgeberischen Eingriffen in die gesetzliche Rentenversicherung. Die wesentlichen Maßnahmen, die die schrittweise Leistungsreduzierung, die Senkung des Rentenniveaus und einen späten Rentenzugang in der Bevölkerung durchgesetzt haben, sind: Anhebung der Altersgrenze auf 65 Jahre für Frauen und Männer (1992/1996); Anhebung der Altersgrenze für schwerbehinderte Menschen auf 63 Jahre (1999); Abschaffung der besonderen Rentenregelungen für Frauen und Arbeitslose (1999); Abschaffung der Rente wegen Berufsunfähigkeit (1999); Flächendeckende Abschläge bei vorzeitigem Rentenbezug seit 2001; Neugestaltung der Rentenformel zur dauerhaften Absenkung des Rentenniveaus (2001); Staatliche Förderung der privaten Altersvorsorge (»RiesterRente«) (2002); Einführung Nachhaltigkeitsfaktor zur dauerhaften Absenkung des Rentenniveaus (2005); Einführung der Rente mit 67 und weitere Kürzung von Rentenansprüchen (2007).

2 Die herangezogenen Befragungsergebnisse beziehen sich auf Betriebe und Beschäftigte aller Branchen und unterschieden nicht zwischen Betrieben mit oder ohne Demografievertrag.

3 Die Erwerbstätigenbefragung von BiBB/BAuA wird bekanntlich in regelmäßigen Zeitabständen wiederholt. Die Datenbasis ist statistisch repräsentativ ausgelegt und die Fragen sind annähernd konstant gehalten. Gradmesser ist das persönliche Empfinden der Beschäftigten, ob eine jeweils spezifische Arbeitsbedingung in ihrer Arbeit häufig auftritt. Bei den Faktoren der Tabelle 1 handelt es sich überwiegend um alternskritische Arbeitsanforderungen.

4 Der Autor hat obige Ergebnisse der Beschäftigtenbefragung des Jahres 2012 mit den Antworten zum subjektiv eingeschätzten Gesundheitszustand aus der gleichen Erhebung in Verbindung gesetzt. Es wird deutlich, dass die Befragten ihren Gesundheitszustand deutlich kritischer einstufen, wenn die genannten körperlichen und psychischen Faktoren auf ihre Arbeitssituation zutreffen.

5 Mit dem Tarifabschluss 2018 für die Beschäftigten der Metall- und Elektroindustrie wurde ein tarifliches Zusatzgeld (T-ZUG) vereinbart. Beschäftigte, die in Schicht arbeiten, Kinder betreuen oder Angehörige pflegen, können wahlweise einen Teil des tariflichen Zusatzgeldes in Zeit umwandeln und dadurch bis zu acht zusätzliche freie Tage im Jahr erhalten.

6 Die Arbeitnehmerüberlassung war bis zum 1. Januar 2003 streng reglementiert. Mit dem Ersten Gesetz für moderne Dienstleistungen am Arbeitsmarkt (»Hartz I«) wurden das besondere Befristungsverbot, das Synchronisationsverbot, das Wiedereinstellungsverbot und die Beschränkung der Überlassungsdauer auf höchstens zwei Jahre aufgehoben. 
$7 \quad 1990$ lag die jahresdurchschnittliche Zahl der Leiharbeitnehmer*innen erstmals über 100.000; bereits acht Jahre später hatte sie sich verdoppelt. Im Zuge der rechtlichen Änderungen im Rahmen der Hartz-Gesetze kam es zu einer weiteren Expansion der Branche. Im November 2017 erreichte die Zahl der Leiharbeitnehmer*innen mit rund 1,08 Millionen ihren vorläufigen Höchststand (Bundesagentur für Arbeit 2020). 
